\title{
The detection of Earth-mass planets around active stars
}

\section{The mass of Kepler-78b}

\begin{abstract}
A. P. Hatzes
Thüringer Landessternwarte Tautenburg, Sternwarte 5, 07778 Tautenburg, Germany

e-mail: artie@tls-tautenburg.de

Received 17 April 2014 / Accepted 28 June 2014

\section{ABSTRACT}

Kepler-78b is a transiting Earth-mass planet in an $8.5 \mathrm{~h}$ orbit discovered by the Kepler Space Mission. We performed an analysis of the published radial velocity measurements for Kepler-78 in order to derive a refined measurement for the planet mass. Kepler-78 is an active star and radial velocity variations due to activity were removed using a floating chunk offset (FCO) method where an orbital solution was made to the data by allowing the velocity offsets of individual nights to vary. We show that if we had no a priori knowledge of the transit period the FCO method, used as a periodogram, would still have detected Kepler-78b in the radial velocity data. It can thus be effective at finding unknown short-period signals in the presence of significant activity noise. Using the FCO method while keeping the ephemeris and orbital phase fixed to the photometric values and using only data from nights where 6-10 measurements were taken results in a $K$-amplitude of $1.34 \pm 0.25 \mathrm{~m} \mathrm{~s}^{-1}$, a planet mass of $1.31 \pm 0.24 M_{\oplus}$, and a planet density of $\rho=4.5_{-2.0}^{+2.2} \mathrm{~g} \mathrm{~cm}^{-3}$. Allowing the orbital phase to be a free parameter reproduces the transit phase to within the uncertainty. The corresponding density implies that Kepler-78b may have a structure that is deficient in iron and is thus more like the Moon. Although the various approaches that were used to filter out the activity of Kepler-78 produce consistent radial velocity amplitudes to within the errors, these are still too large to constrain the structure of this planet. The uncertainty in the mass for Kepler-78b is large enough to encompass models with structures ranging from Mercury-like (iron enriched) to Moon-like (iron deficient). A more accurate $K$-amplitude as well as a better determination of the planet radius are needed to distinguish between these models.
\end{abstract}

Key words. stars: individual: Kepler 78 - techniques: radial velocities - planetary systems

\section{Introduction}

Kepler-78b is a transiting Earth-sized planet in a $0.355-\mathrm{d}$ orbit found by the NASA Kepler Spacecraft (Sanchis-Ojeda et al. 2013). Although a careful analysis of the exquisite Kepler light curves by Sanchis-Ojeda et al. (2013) established with high probability that the transiting object was planetary in nature, final confirmation required Doppler measurements. This was established simultaneously by two teams.

Howard et al. (2013, hereafter H2013) took radial velocity (RV) measurements using the HIRES spectrograph on the Keck telescope. They employed a version of harmonic analysis where a series of sine functions were used to fit the RV signal due to activity. The periods of the sine functions were restricted to the rotation period of the star, $P_{\text {rot }}$ and its harmonics $\left(P_{\text {rot }} / 2\right.$, $P_{\text {rot }} / 3$, etc.). The derived planet mass was $1.69 \pm 0.41 M_{\oplus}$ (velocity $K$-amplitude $=1.66 \pm 0.40 \mathrm{~m} \mathrm{~s}^{-1}$ ). This planet bulk density was $5.3_{-1.6}^{2.0} \mathrm{~g} \mathrm{~cm}^{-3}$ using a planet radius of $r_{\mathrm{p}}=1.20 \pm 0.09 R_{\oplus}$.

Pepe et al. (2013, hereafter P2013) used RV measurements from the HARPS-N spectrograph to measure a $K$-amplitude of $1.96 \pm 0.32 \mathrm{~m} \mathrm{~s}^{-1}$, also based on harmonic analysis of the rotation period. This resulted in a planet mass of $m_{\mathrm{p}}=1.86_{-0.25}^{0.38} M_{\oplus}$ and a planet density of $\rho_{m}=5.57_{-1.31}^{3.02} \mathrm{~g} \mathrm{~cm}^{-3}$ using a planet radius of $r_{\mathrm{p}}=1.173_{-0.089}^{0.159} R_{\oplus}$. P2013 also employed the technique where the orbit was fit by allowing the nightly velocity offset to vary (see below). This technique was first used to determine the mass of the transiting planet, CoRoT-7b (Hatzes et al. 2010). P2013 derived a velocity $K$-amplitude of $2.08 \pm 0.32 \mathrm{~m} \mathrm{~s}^{-1}$, a value consistent with the harmonic analysis of the same data set and the value of H2013. Both density determinations firmly established Kepler-78b as a rocky planet. The remarkable aspect of the RV detection of the planet was the fact that the host star, Kepler-78, is modestly active star showing activity RV "jitter" of $\approx \pm 10 \mathrm{~m} \mathrm{~s}^{-1}$. The activity signal thus dominates the RV reflex motion caused by the planet by a factor five or more.

The RV detection of Kepler-78 b was made possible because ultra-short period planets offer us a way to extract their signals from RV data that are plagued by stellar activity noise. If the orbital period of the planet is much smaller than the rotation period of the star, the nominal timescale of activity, it is possible to detect the planetary signal. Kepler-78b has an orbital period of mere $8.5 \mathrm{~h}$, much smaller than the stellar rotational period of $12.8 \mathrm{~d}$ (H2013). Extracting short period planet signals from RV data of active stars was first demonstrated for CoRoT-7b, a planet with a $0.85 \mathrm{~d}$ period orbiting an active star whose rotation period is $23 \mathrm{~d}$ (Queloz et al. 2009; Hatzes et al. 2010).

In this paper we present an independent analysis of all the available RV data for Kepler-78. Our goals are:

1. To determine a refined mass using the combined HARPS-N and Keck RV measurements.

2. To assess the robustness of the detected planets to different approaches used in filtering out the activity signal.

3. To assess whether a Kepler-78b-like exoplanet can be detected in the RV data and accurate orbital elements derived without the transit information. 


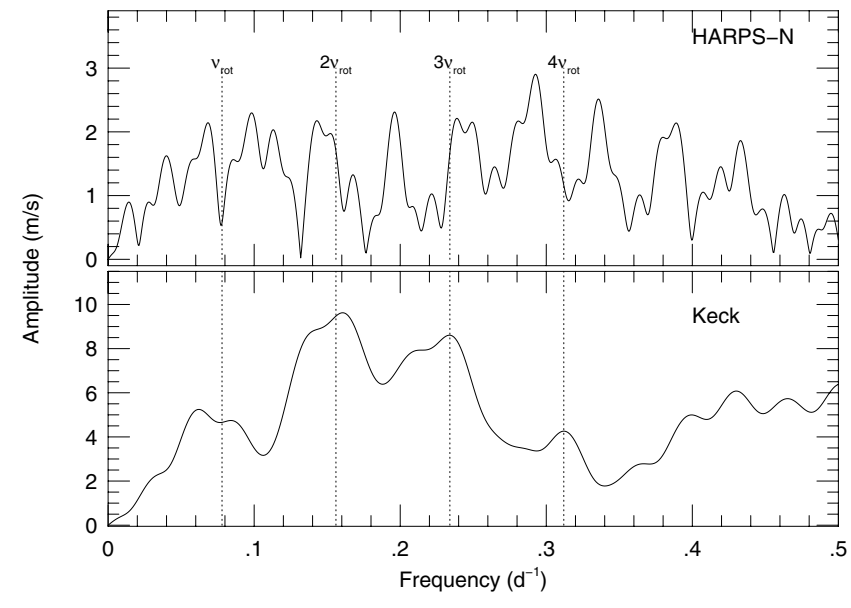

Fig. 1. Top: low frequency Fourier amplitude spectrum of the HARPS-N RV data. The dashed vertical lines mark the location (left to right) of the rotational frequency, $v_{\text {rot }}$ and its first three harmonics $\left(2 v_{\mathrm{rot}}\right.$, $\left.3 v_{\text {rot }}, 4 v_{\text {rot }}\right)$. Bottom: same for the Keck RV data.

\section{Period analysis}

\subsection{Prewhitening}

Fourier component analysis via the so-called prewhitening procedure is a standard way of extracting multi-periodic signals from time series data. It selectively identifies the Fourier components of a time series by first finding the dominant frequency, fitting a sine wave to the data and removing this, and then searching for the next highest Fourier component. An obvious application is the detection of multi-periodic planet systems (e.g. Hatzes 2013a), but it can also be an effective tool for filtering out activity noise that may not be strictly periodic (Hatzes et al. 2010, $2013 b$ ). We should note that harmonic analysis based on the stellar rotation frequency is a form of prewhitening. However, it restricts the choice of frequencies only to that of rotational frequency and its harmonics. With traditional prewhitening, on the other hand, one can select the highest amplitude frequency found in the time series or its residuals, even if it is not obviously related to the rotation of the star.

We applied prewhitening individually to the HARPS-N and Keck data sets in order to evaluate the Fourier spectrum of each time series and to test how effective this procedure is in extracting the planetary signal. All data values were used, including nights where only one RV measurement was made.

Figure 1 shows the low frequency Fourier amplitude spectrum of each data set centered around the expected stellar rotational frequency. Vertical dashed lines mark the location of the rotational frequency, $v_{\text {rot }}$, as well as its first 3 harmonics $\left(2 v_{\text {rot }}\right.$, $3 v_{\text {rot }}$, and $\left.4 v_{\text {rot }}\right)^{1}$. Note that the Keck RV data are clearly dominated by the first harmonic, but it also shows strong evidence for the presence of $3 v_{\text {rot }}$. The rotational frequency and $4 v_{\text {rot }}$ are also present, but only weakly so. The Fourier amplitude spectrum lends support to the use of harmonic analysis on the Keck data. $\mathrm{H} 2013$ only used components out to $3 v_{\text {rot }}$ since including higher terms did not improve the solution. This is also supported by the Fourier spectrum as the amplitude of $4 v_{\text {rot }}$ significantly drops from that of $3 v_{\text {rot }}$.

The HARPS-N data, on the other hand, show no clear evidence for the rotational frequency or any of the harmonics. The

\footnotetext{
1 The convention for this paper is to start the numbering of the harmonics with the first "overtone" $2 v_{\text {rot }}\left(P_{\text {rot }} / 2\right)$, rather than with the rotational frequency.
}

Table 1. HARPS-N prewhitened frequencies and amplitudes.

\begin{tabular}{lccc}
\hline \hline $\begin{array}{l}\text { Period } \\
(\mathrm{d})\end{array}$ & $\begin{array}{c}\text { Amplitude } \\
\left(\mathrm{m} \mathrm{s}^{-1}\right)\end{array}$ & Phase & Comment \\
\hline $3.425 \pm 0.019$ & $3.60 \pm 0.39$ & $0.837 \pm 0.015$ & $\approx P_{\text {rot }} / 4$ \\
$10.405 \pm 0.089$ & $4.26 \pm 0.43$ & $0.185 \pm 0.013$ & $\approx P_{\text {rot }}$ \\
$0.3549 \pm 0.003$ & $1.88 \pm 0.44$ & $0.729 \pm 0.029$ & $P_{\mathrm{K} 78 \mathrm{~b}}$ \\
$6.2578 \pm 0.480$ & $1.62 \pm 0.48$ & $0.035 \pm 0.034$ & $P_{\text {rot }} / 2$ \\
\hline
\end{tabular}

Notes. $P_{\text {rot }}$ is the rotation period of the star and $P_{\mathrm{K} 78}$ is the orbital period of Kepler-78b. The phase is reckoned with respect to the time of the first HARPS-N measurement.

Table 2. Keck prewhitened frequencies and amplitudes.

\begin{tabular}{lccc}
\hline \hline $\begin{array}{l}\text { Period } \\
\text { (d) }\end{array}$ & $\begin{array}{c}\text { Amplitude } \\
\left(\mathrm{m} \mathrm{s}^{-1}\right)\end{array}$ & Phase & Comment \\
\hline $6.443 \pm 0.0370$ & $11.92 \pm 0.45$ & $0.48 \pm 0.013$ & $P_{\text {rot }} / 2$ \\
$4.235 \pm 0.0897$ & $9.67 \pm 0.62$ & $0.12 \pm 0.014$ & $P_{\text {rot }} / 3$ \\
$0.3549 \pm 0.0024$ & $2.01 \pm 0.43$ & $0.68 \pm 0.037$ & $P_{\text {K78b }}$ \\
$11.312 \pm 0.171$ & $3.66 \pm 0.41$ & $0.31 \pm 0.029$ & $P_{\text {rot }}$ \\
\hline
\end{tabular}

Notes. The phase is reckoned with respect to the time of the first Keck measurement.

dominant frequency at $v=0.29 \mathrm{~d}^{-1}$ might be related to $4 v_{\text {rot }}$, which implies a rotational period of $13.8 \mathrm{~d}$, much higher than the rotational period found by $\mathrm{H} 2013$.

Tables 1 and 2 list the frequencies found by the prewhitening process for the HARPS-N and Keck data, respectively. Both produce comparable rms scatter about the fit: $2.57 \mathrm{~m} \mathrm{~s}^{-1}$ for HARPS-N and $2.51 \mathrm{~m} \mathrm{~s}^{-1}$ for Keck. We can exploit the fact that the Keck data clearly show evidence for the presence of rotational harmonics to determine the rotational period. If the "nonplanetary" frequencies are indeed due to rotational harmonics then the observed low frequencies should occur at intervals of $v_{\text {obs }}=n v_{\text {rot }}$, where $v_{\text {rot }}$ is the rotational frequency and $n$ an integer. The value of $v_{\text {rot }}$ that minimized $\sum_{n=1}^{3}\left(v_{\text {obs }}-n v_{\text {rot }}\right)^{2}$ corresponded to $P_{\text {rot }}=12.7 \pm 0.3 \mathrm{~d}$, consistent with the value of H2013.

What is surprising is that pre-whitening of the HARPS-N data produced essentially the same $K$-amplitude even though standard prewhitening found differerent frequencies. The $10.4 \mathrm{~d}$ period is near the $\sim 10 \mathrm{~d}$ period used by P2013, whereas the $3.42 \mathrm{~d}$ period is close to, but not coincident with the $4.2 \mathrm{~d}$ period used by $\mathrm{P} 2013$. The $6.2 \mathrm{~d}$ period found by pre-whitening was not mentioned by P2013. Regardless of the fact that different Fourier components for the activity signal have been removed, the same amplitude is derived: $1.88 \pm 0.44 \mathrm{~m} \mathrm{~s}^{-1}$ for prewhitening compared to $1.96 \pm 0.32 \mathrm{~m} \mathrm{~s}^{-1}$ for harmonic analysis. This speaks for the robustness of the Kepler-78b detection, an issue that we shall return to later.

An orbital solution using the transit ephemeris and zero eccentricity was made to the combined residual data after removing the activity signal (i.e. frequencies) from the data. The solution resulted in a $K$-amplitude of $1.90 \pm 0.23 \mathrm{~m} \mathrm{~s}^{-1}$ which is consistent with both P2013 and H2013.

\subsection{The floating chunk offset periodogram}

The RV detection of Kepler-78b was abetted by the fact that a periodic transit signal had been found in the photometry that was validated as due to an orbiting planet (Sanchis-Ojeda et al. 2013). We thus have the luxury of knowing both the orbital 


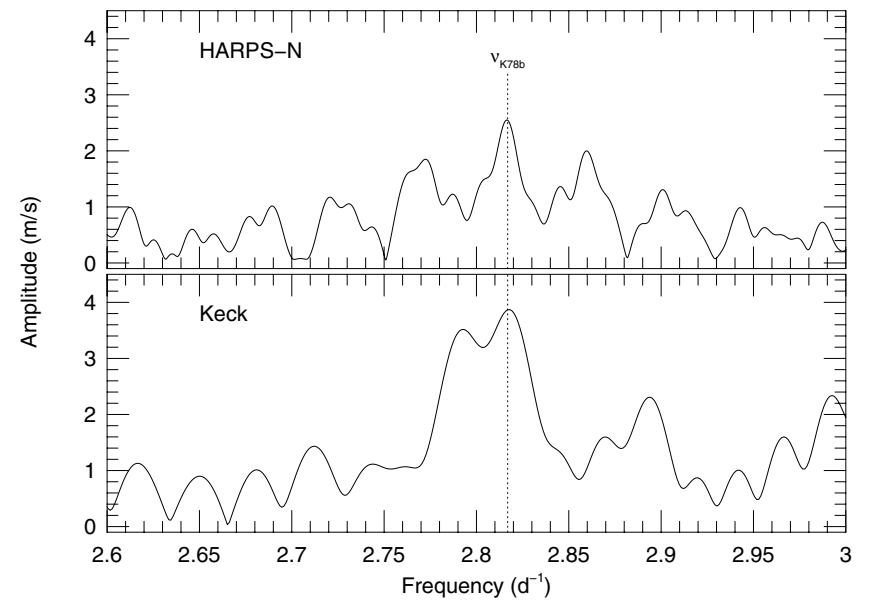

Fig. 2. Top: Fourier amplitude spectrum of the HARPS-N RV data centered on the orbital frequency of Kepler-78b $\left(v_{\mathrm{K} 78 \mathrm{~b}}\right.$, dashed vertical line). Bottom: same for the Keck RV data.

period and orbital phase - we only need to fit the velocity amplitude. The question naturally arises, "Could Kepler-78b had detected without a priori knowledge of a transiting planet?"

There is already evidence in the raw Fourier amplitude spectrum for the presence of Kepler-78b. These show what appears to be a significant peak precisely at the orbital frequency of Kepler-78b (Fig. 2). In both the HARPS-N and Keck data this peak is approximately four times the height of the surrounding noise floor. Kuschnig et al. (1997) established through simulations that this corresponds to a false alarm probability of about $1 \%$. The planet signal is reasonably significant without prior knowledge of the transit period. However, in the case of active stars the Fourier spectrum can be completely dominated by the numerous frequencies arising from activity that make it difficult to assess the true statistical significance.

Hatzes et al. (2010) introduced a method by which one could extract the signal of short period planets even in the presence of considerable activity noise. The method exploits cases where the planet orbital period is significantly less than periods arising from activity. Depending on the rotational period of the star, during one night the RV contribution by surface structure (spots, plage, etc.) is small since the star has had little time to rotate, or even for spots to evolve. The spot configuration on the star is essentially frozen-in and it only adds a constant value to the observed RV. When taking a long time series of measurements throughout the night one should only see changes in the RV due to the orbital motion of the planet. Fitting these RV measurements with a Keplerian orbit, but allowing the zero points of the nightly data to vary is an effective way to filter out the activity variations and to derive an accurate measurement of the $K$-amplitude. We shall refer to this technique as the floating chunk offset (FCO) method.

FCO was successfully applied to the first transiting rocky planet CoRoT-7b, a case where the $K$-amplitude of the planet was 2-4 times lower than the variations due to activity (Hatzes et al. 2010, 2011). In the case of Kepler-78b we are hindered by the fact that the $K$-amplitude is a factor of 5-10 times lower than the activity signal, but we are helped by a much shorter orbital period for Kepler-78b (0.355 d compared to $0.85 \mathrm{~d}$ for CoRoT-7b).

FCO can also be used as a form of a "periodogram" (Hatzes et al. 2010, 2011) to search for planetary signals in the presence of activity noise. For a transiting planet the orbital period

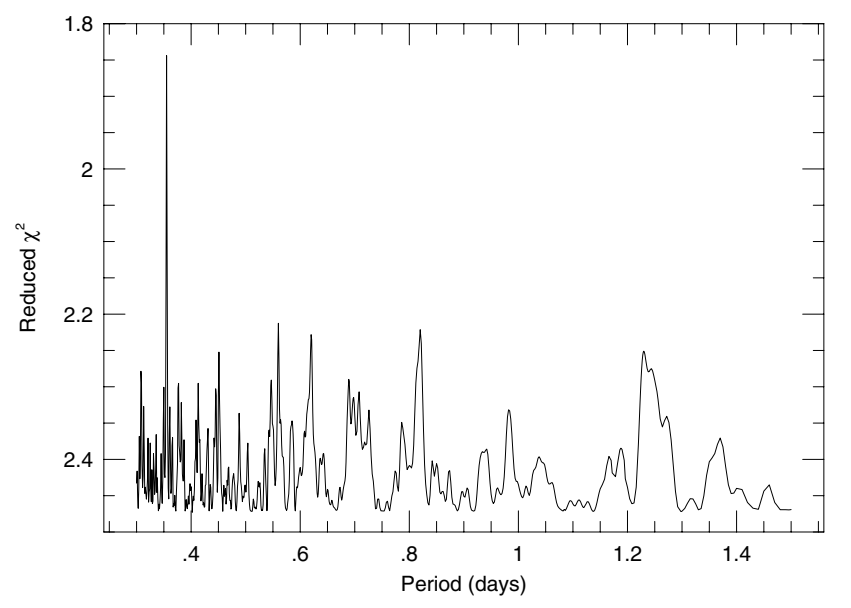

Fig. 3. FCO periodogram of the combined Kepler-78 RVs (HARPS-N plus Keck). The scale of the $y$-axis has been reversed so that a lower $\chi^{2}$ appears as a peak.

is known, so a periodogram is of limited use, except possibly for investigating the signficance of the signal. The true utility of a periodogram, however, is finding unknown planets in your time series. For these we do not know the phase of the orbit (e.g. transit time), the period, or the eccentricity.

To explore the behavior of the FCO as a "periodogram" we fitted the data using a trial orbital period and allowed the nightly offsets to vary. The trial period was then varied over a range of values and the goodness-of-fit examined. The eccentricity was fixed to zero since circular orbits are a good assumption for short period planets. However, it is possible to vary the eccentricity once a best-fit period is found. We also allowed the orbital phase to vary because another period with a different phase might provide a better fit to the data than using the transit phase. For each solution we computed the reduced $\chi^{2}$ and then repeated this for another trial period. The lowest value of $\chi^{2}$ in the $\chi^{2}$-period diagram revealed the strong periodic signal present in the data. The FCO fit was performed using the least squares fitting program Gaussfit (Jefferys et al. 1988).

Figure 3 shows this FCO periodogram as applied to combined Kepler-78b RV data. Note that even though the Keck and HARPS-N have different zero point offsets, this is irrelevant to FCO as it calculates velocity offsets for separate nights. It thus offers a natural way of combining data taken with different instruments and velocity offsets in a least squares sense. In this figure the scale of the $y$-axis is reversed so as to mimic a classic periodogram where a peak signifies high power, and thus a signifcant signal. In our case the peak of the FCO periodogram is not a power, but goodness of fit. We plot $\chi^{2}$ as opposed to $1 / \chi^{2}$ so that the reader can get an immediate sense for the quality of the fit. The $\chi^{2}$ shows a clear peak (minimum) at the orbital period of Kepler-78b.

\subsection{Tests of the FCO periodogram}

The FCO method was tested on simulated activity signals to see how well it could recover a known planet signal inserted into the data. To do this we added the Kepler- $78 \mathrm{~b}$ orbit to simulated RV data sets generated by two activity "models". The first (Model 1) consisted of an activity signal generated using the frequencies found in the prewhitening process. The respective prewhitened frequencies for each data set were used (Table 1 for the "HARPS-N" data and Table 2 for the "Keck" data), but 


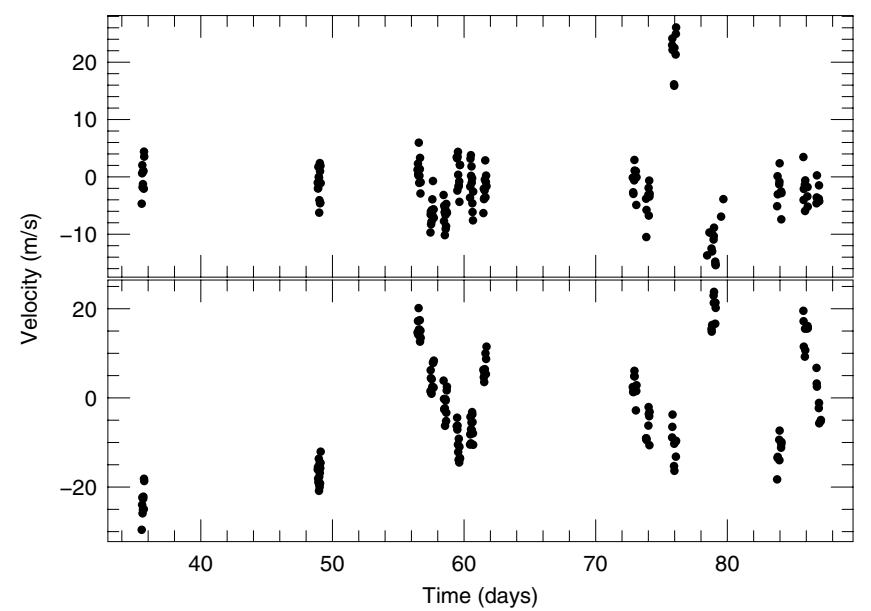

Fig. 4. Top: simulated data consisiting of a simulated activity signal (Model 1), a planet signal, plus random noise with $\sigma=2.4 \mathrm{~m} \mathrm{~s}^{-1}$. Bottom: simulated data using Model 2 for the activity signal.

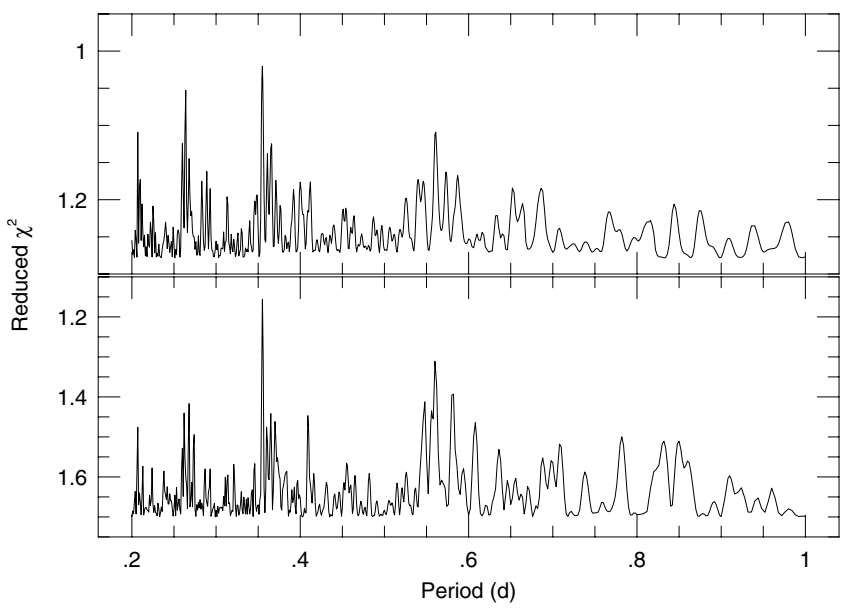

Fig. 5. Top: FCO periodogram of the simulated data set from Model 1 for the activity plus planet. Bottom: FCO periodogram for Model 2 plus planet.

without the planet signal. The orbital solution was then inserted into the activity signal with $K=1.5 \mathrm{~m} \mathrm{~s}^{-1}$. These simulated data were sampled the same way as the real data and random noise at a level of $\sigma=2.4 \mathrm{~m} \mathrm{~s}^{-1}$ was also added. The top panel of Fig. 4 shows the simulated data set.

The resulting FCO periodogram is shown in the top panel of Fig. 5. Again, in this case we also allowed the orbital phase for each trial period to vary. The highest peak (minimum $\chi^{2}$ ) corresponds to the planet frequency. The top panel of Fig. 6 shows the Lomb-Scargle (L-S) periodogram (Lomb 1976; Scargle 1982) of the original data, We use the L-S periodogram in its original form rather than the generalized L-S periodogram (GLS, Zechmeister \& Kürster 2009) because we prefer to show the unnormalized power - the larger the power, the higher the significance of a peak. For data sets with a large number of points the L-S periodogram produces consistent results as GLS. Note that one clearly see the signal of the planet in the unfiltered L-S periodogram in spite of the activity signal. The lower panel shows the L-S periodogram after applying the nightly offsets from the best fit period. The offset fitting has acted as a high pass filter that has suppressed the low frequency components due to activity and greatly increased the power at the planet orbital frequency. The recovered $K$-amplitude was $K=1.29 \pm 0.26 \mathrm{~m} \mathrm{~s}^{-1}$.

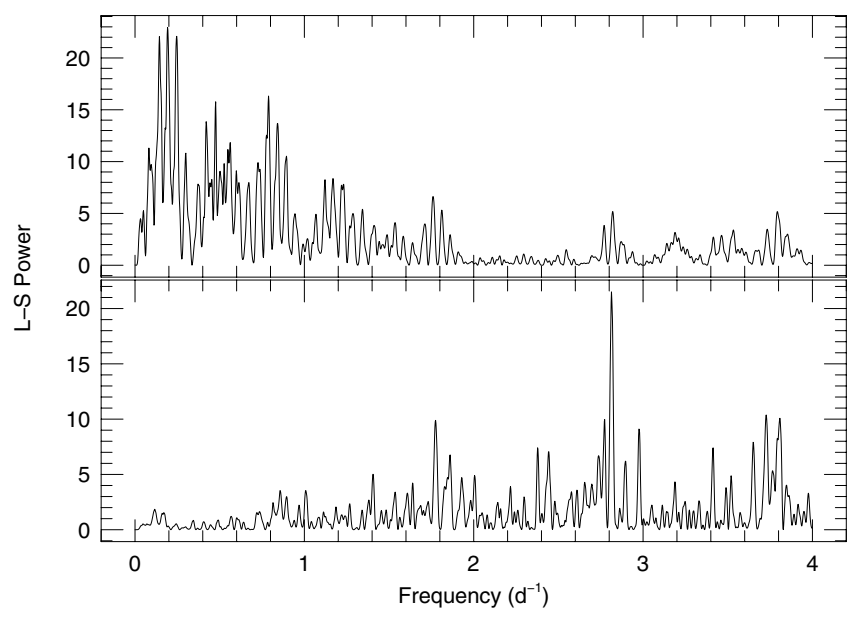

Fig. 6. Top: L-S periodogram of the simulated Model 1 data. Bottom: L-S perodogram of simulated Model 1 data after removing the calculated nightly offsets.

For the second model of the activity variations we considered a more complicated case. The times of the data were divided into three epochs: JD $-2440000=35-49,56-61$, and 72-87. In each epoch we generated a simulated activity signal using a sum of sine functions but with slightly different rotation periods and harmonics in each epoch. The periods and amplitudes for the sine functions were as follows. Epoch 1: $P_{1}=12.83 \mathrm{~d}$, $K_{1}=20.7 \mathrm{~m} \mathrm{~s}^{-1} ; P_{2}=6.42 \mathrm{~d}, K_{2}=10.6 \mathrm{~m} \mathrm{~s}^{-1}$. Epoch 2: $P_{1}=11.50 \mathrm{~d}, K_{1}=9.0 \mathrm{~m} \mathrm{~s}^{-1} ; P_{2}=6.42 \mathrm{~d}, K_{2}=15.0 \mathrm{~m} \mathrm{~s}^{-1}$; $P_{3}=3.70 \mathrm{~d}, K_{3}=4.6 \mathrm{~m} \mathrm{~s}^{-1}$. Epoch 3: $P_{1}=13.61 \mathrm{~d}$, $K_{1}=5.0 \mathrm{~m} \mathrm{~s}^{-1} ; P_{2}=6.8 \mathrm{~d}, K_{2}=10.0 \mathrm{~m} \mathrm{~s}^{-1} ; P_{3}=3.44 \mathrm{~d}$, $K_{3}=8.6 \mathrm{~m} \mathrm{~s}^{-1}$. Note that in each epoch slightly different "rotational" periods and harmonics were used. This was to mimic spots located and emerging at different latititudes of a differentially rotating star. We should point out this might present difficulties to the harmonic analysis which assumes a single rotation period and its harmonics.

The lower panel of Fig. 4 shows the resulting simulated activity signal. This includes the planet signal as well as random noise with $\sigma=2.4 \mathrm{~m} \mathrm{~s}^{-1}$. Notice that this model produces much larger variations for the activity signal, $\Delta \mathrm{RV}= \pm 20 \mathrm{~m} \mathrm{~s}^{-1}$. The resulting FCO periodogram is shown in the lower panel of Fig. 5. Again, the $\chi^{2}$ is minimized (appearing as the highest peak) for the correct orbital period. The fitted $K$-amplitude was $K=1.48 \pm 0.27 \mathrm{~m} \mathrm{~s}^{-1}$.

Figure 7 shows the L-S periodogram of the original (top) and filtered (bottom) RV data. Note that there is virtually no power at the orbital frequency of $2.817 \mathrm{~d}^{-1}$ in the original data, but that this becomes very prominent in the power spectrum of the filtered data. Again, the numerous peaks at low frequency due to activity are effectively suppressed.

As a final check of the robustness of the planet signal we took the Keck + HARPS RV data and applied the chunk offset fitting method, but used the wrong orbital period, i.e. a period other than $0.355-\mathrm{d}$, in calculating the offsets. Two trial periods were used in the fitting, the second and third minima (peaks) in the FCO periodogram, namely $P=0.56 \mathrm{~d}$ and $0.82 \mathrm{~d}$. Figure 8 shows the L-S periodogram of the RV data after removing the nightly offsets calculated with the incorrect orbital periods. Remarkably, the true orbital frequency at $2.817 \mathrm{~d}^{-1}$ still comes through as the dominant peak. In the case of the $0.56 \mathrm{~d}$ fit, it is clearly the dominant peak. In the 0.82-d fit, it is comparable to the spurious peak at the frequency corresponding 


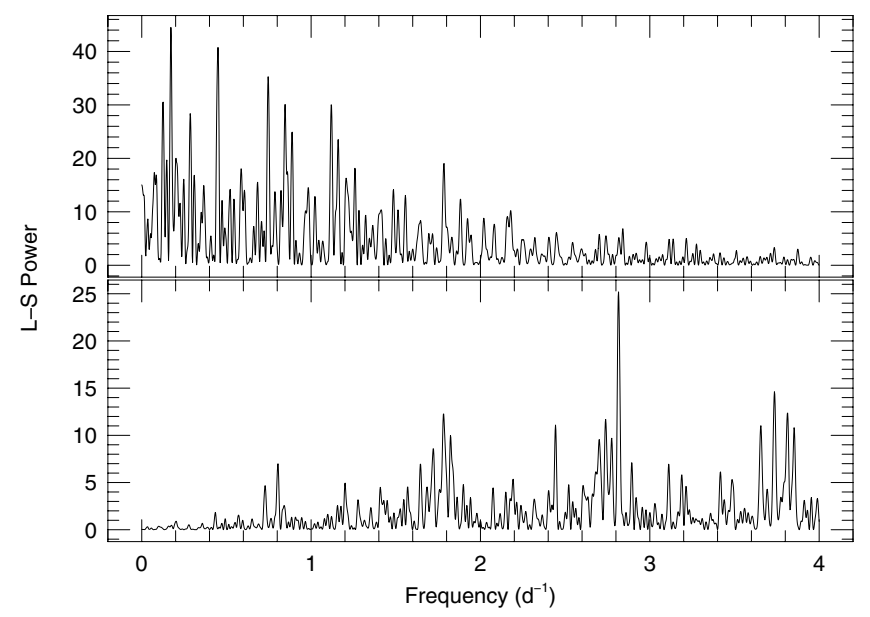

Fig. 7. Top: L-S periodogram of the simulated Model 2 data. Bottom: L-S perodogram of simulated Model 2 data after removing the calculated nightly offsets.

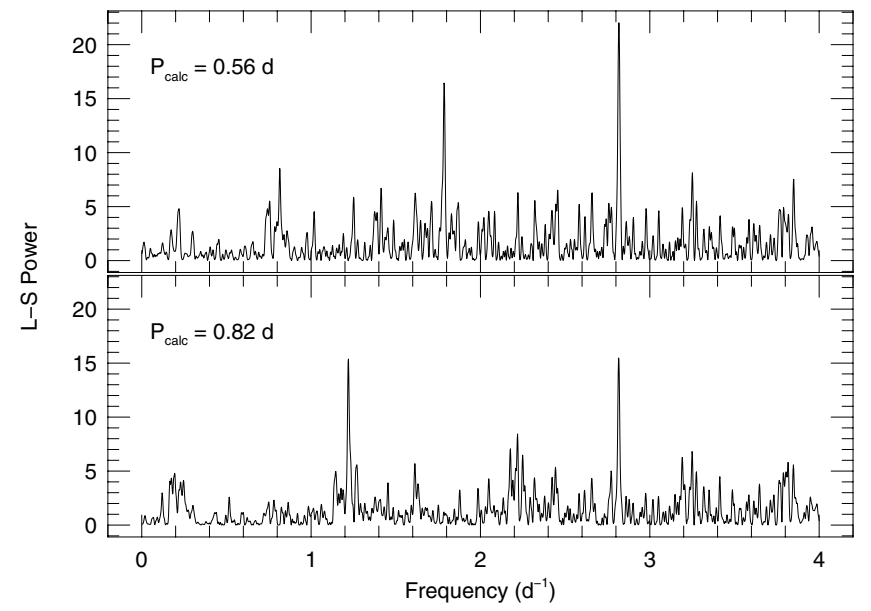

Fig. 8. L-S periodogram after using the FCO method on the RV data with a period of $0.56 \mathrm{~d}($ top $)$ and $0.82 \mathrm{~d}$ (bottom). Note that the orbital frequency of Kepler-78b $\left(v=2.817 \mathrm{~d}^{-1}\right)$ is still the dominant peak.

to the period $(0.82-\mathrm{d})$ used in calculating the nightly offsets. This speaks for the robustness of the planet signal found by the FCO method.

To explore the detection limit of a Kepler-78b-like planet in the RV data, we took the first activity model (Model 1) as it best represents the variations seen in the actual data. We then added the planet signal with $K=1.0 \mathrm{~m} \mathrm{~s}^{-1}$ and noise at a level of $2.4 \mathrm{~m} \mathrm{~s}^{-1}$. Figure 9 shows the resulting FCO periodogram. It is considerably noiser, but the dominant peak still occurs at the planet frequency.

The top panel of Fig. 10 shows the L-S periodogram after fitting these synthetic data with the $0.355-\mathrm{d}$ period and subtracting the computed offsets. The dominant peak is at the correct orbital frequency for the planet. We also show two other periodograms where in these cases we calculated the nightly offsets using the wrong "orbital" periods of $0.264 \mathrm{~d}$ and $0.586 \mathrm{~d}$. These periods coincide with local minima (i.e. peaks in the periodogram). The dominant peak remains at the true value of $v=2.817 \mathrm{~d}^{-1}$.

\section{The mass of Kepler-78b}

An orbital fit was made to the combined RV data using the FCO technique in order to get a better determination of the

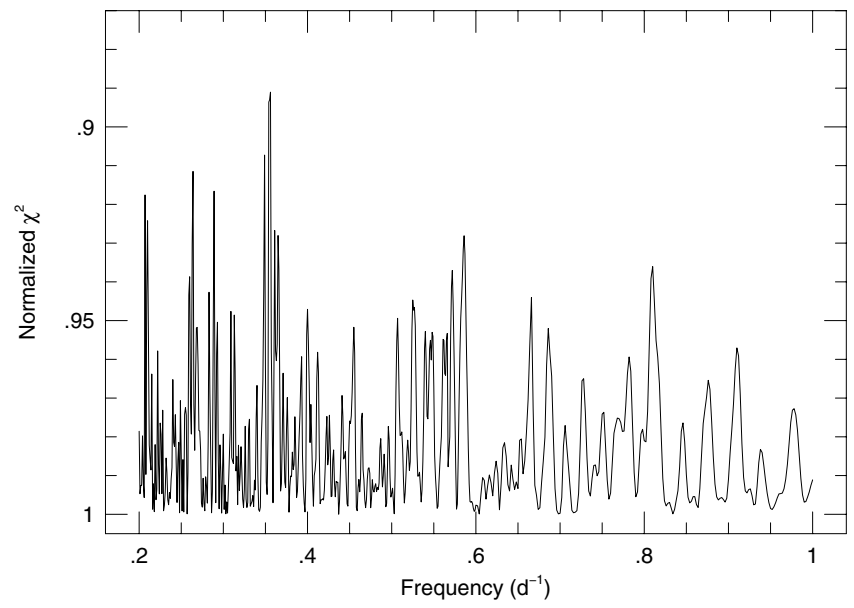

Fig. 9. FCO periodogram using the second activity model and the planet orbit added with $K=1.0 \mathrm{~m} \mathrm{~s}^{-1}$. The highest peak coincides to the orbital frequency of $v=2.817 \mathrm{c} \mathrm{d}^{-1}$.

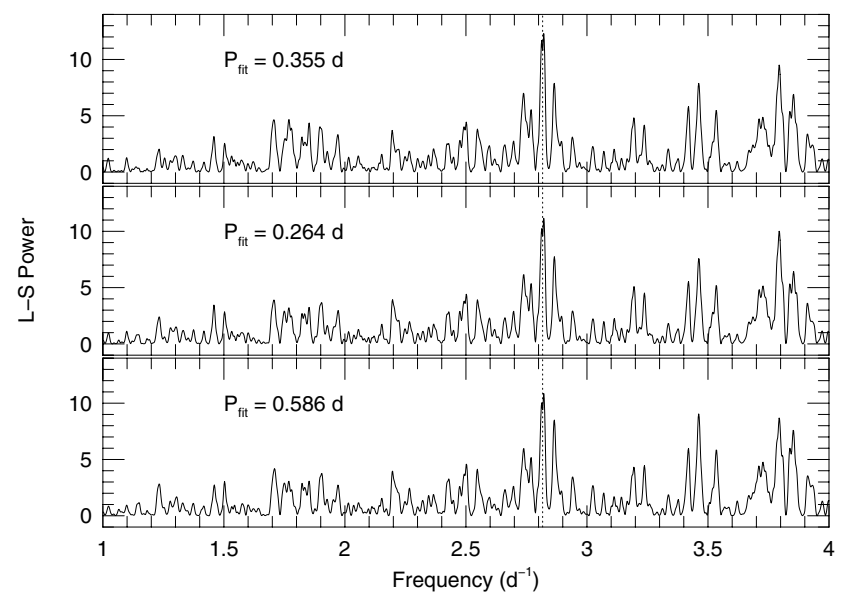

Fig. 10. L-S periodogram of the simulated data with a planet $K$ amplitude of $1.0 \mathrm{~m} \mathrm{~s}^{-1}$ and after subtracting the calculated offsets. (Top) Offsets calculated using the true planet orbital period. The lower panels show the results after using the wrong orbital periods for calculating the offsets, $0.264 \mathrm{~d}$ (middle) and $0.586 \mathrm{~d}$ (bottom). The dominant peak is still at the true orbital frequency.

$K$-amplitude. Three approaches were made to fitting the data: 1) the data in its original form and the estimated errors; 2) trend fitting on nightly data with the addition of a so-called jitter correction; and 3) fitting only those nights for which multiple measurements $(N>2$, but typically $6-10)$ were made.

First, an FCO fit was made to the individual data sets keeping the ephemeris (epoch of transit and orbital phase) fixed to the values derived from the transit light curve. The HARPS-N (H-N) only data resulted in $K=1.86 \pm 0.30 \mathrm{~m} \mathrm{~s}^{-1}$, observed minus computed $(\mathrm{O}-\mathrm{C})=2.11 \mathrm{~m} \mathrm{~s}^{-1}$, and a reduced $\chi^{2}=1.03$ (Solution 4 in Table 3). These values are fully consistent with those published by P2013.

We also investigated allowing the orbital phase to be a free parameter. If the derived orbital phase is consistent with the transit values this would provide even stronger confirmation that the RV variations are indeed due to a planet. For circular orbits the angle of periastron, $\omega$, is ill-defined, but it can still be used as a measure of the orbital phase. For eclising systems where the epoch is defined as the primary eclipse (mid-transit time in our case) it should be exactly $90^{\circ}$ (see Phoebe 2.0.0 
Table 3. $K$-ampitudes from the different solutions, $N$.

\begin{tabular}{lccccc}
\hline \hline$N$ & $\begin{array}{c}K \\
\left(\mathrm{~m} \mathrm{~s}^{-1}\right)\end{array}$ & $\begin{array}{c}\mathrm{O}-\mathrm{C}_{H} \\
\left(\mathrm{~m} \mathrm{~s}^{-1}\right)\end{array}$ & $\begin{array}{c}\mathrm{O}-\mathrm{C}_{K} \\
\left(\mathrm{~m} \mathrm{~s}^{-1}\right)\end{array}$ & $\chi^{2}$ & Comment \\
\hline 1 & $1.96 \pm 0.32$ & 2.34 & - & - & P2013 \\
2 & $1.66 \pm 0.40$ & - & - & 1.12 & H2013 \\
3 & $1.90 \pm 0.32$ & 2.59 & 2.55 & 2.60 & Prewhitened \\
4 & $1.86 \pm 0.30$ & 2.11 & 2.55 & 1.03 & H-N \\
5 & $1.72 \pm 0.40$ & - & 2.58 & 2.49 & Keck \\
6 & $1.77 \pm 0.26$ & - & 2.58 & 2.49 & All, no Jitter \\
7 & $1.63 \pm 0.23$ & 2.12 & 2.58 & 1.02 & All w/Jitter \\
8 & $1.30 \pm 0.35$ & - & 2.58 & 1.00 & Keck w/Jitter \\
9 & $1.39 \pm 0.37$ & - & 2.08 & 0.95 & H-N, $N>2$ \\
10 & $1.34 \pm 0.25$ & 2.08 & 2.59 & 0.95 & Adopted \\
\hline & & & & & \\
\hline
\end{tabular}

documentation $)^{2}$. So, when fitting the orbit to a fixed epoch, the phase of the orbit is represented by the parameter $\omega$. In this case, a value of $\omega$ significantly different from $90^{\circ}$ would indicate that the RV data are better fit with a circular orbit not in phase with the time of mid-transit and this would cast some doubt on the reality of the RV signal.

Allowing the orbital phase to vary resulted in $\omega=88.6 \pm$ 11.9 degrees, a value consistent with the transit ephemeris and strong confirmation that the RV variations are due to the planet. Had one discovered Kepler-78b with RV measurements first, the HARPS-N data are of sufficient quality to predict the time of transit to within 0.035 orbital phase or $18 \mathrm{~min}$.

The fit using only the Keck data and again fixing the orbital phase to the transit value resulted in $K=1.72 \pm 0.40 \mathrm{~m} \mathrm{~s}^{-1}$, $\mathrm{O}-\mathrm{C}=2.58 \mathrm{~m} \mathrm{~s}^{-1}$, and $\chi^{2}=2.49$ (Solution 5 in Table 3 ). These values are consistent with those of $\mathrm{H} 2013$. Allowing the orbital phase to be a free parameter resulted in $\omega=74.6 \pm 16.7$ degrees, again in agreement with the transit ephemeris to $1 \sigma$. Given the short orbital period the Keck solution could predict the transit time to within $20 \mathrm{~min}$.

Finally, fitting all the Keck + HARPS-N RV data yielded $K=1.77 \pm 0.26 \mathrm{~m} \mathrm{~s}^{-1}$ (Solution 6).

H2013 noted that on some nights the Keck data had linear trends that were removed before computing the orbit. We performed a visual inspection to the data and noticed that only on Night 4 (JD $-2456400=75.8-76.10)$ was there a significant linear trend in the data. The other nights showed only marginal trends that were difficult to discern due to the scatter of the measurements. This was confirmed by performing a linear least squares fit to the nightly data. On Night 4 the slope in the RV variations was more than four times larger than its error. On all other nights the slope in the RV variations had a value of zero to within the errors. Therefore, for the other nights we chose not to detrend the data as these might adversely affect the $K$-amplitude. The Keck data typically cover around 0.3 of an orbital phase and during this time the orbital motion itself might appear as a linear trend. The same, of course, holds for Night 4 , however the trend in this case was large $\left(\triangle \mathrm{RV}=5 \mathrm{~m} \mathrm{~s}^{-1}\right)$ and all measurements during the night followed the linear trend. We can thus be reasonably confident of fitting mostly the variations of the trend and not suppressing the variations due to the orbital motion.

H2013 also increased the measurement error by applying a "jitter" correction to account for the actual errors being larger than the estimated ones. The fact that the reduced $\chi^{2}$ of the Keck solution is much larger than unity (Table 3 ) indicates that there may be additional sources of error in the Keck measurements.

\footnotetext{
2 http://www.phoebe-project.org
}

Table 4. Jitter values for the Keck data.

\begin{tabular}{lccc}
\hline \hline $\begin{array}{l}\text { Dates } \\
(\mathrm{JD}-2456400)\end{array}$ & $\begin{array}{c}\text { Mean error } \\
\left(\mathrm{m} \mathrm{s}^{-1}\right)\end{array}$ & $\begin{array}{c}\mathrm{O}-\mathrm{C} \\
\left(\mathrm{m} \mathrm{s}^{-1}\right)\end{array}$ & $\begin{array}{c}\text { Jitter } \\
\left(\mathrm{m} \mathrm{s}^{-1}\right)\end{array}$ \\
\hline $48.88-49.11$ & 1.95 & 2.87 & 2.10 \\
$72.82-73.10$ & 1.89 & 2.63 & 1.83 \\
$73.83-74.10$ & 1.69 & 3.22 & 2.74 \\
$75.80-66.11$ & 1.64 & 1.68 & 0.00 \\
$78.80-79.12$ & 1.60 & 2.43 & 1.83 \\
$83.79-84.12$ & 1.74 & 3.12 & 2.59 \\
$85.78-86.10$ & 1.64 & 3.15 & 2.69 \\
$86.78-87.11$ & 1.74 & 1.92 & 0.81 \\
\hline
\end{tabular}

H2013 applied an overall jitter correction of $2.1 \mathrm{~m} \mathrm{~s}^{-1}$ that was added in quadrature to the nominal errors. The source of this jitter is not clear, but it most likely due to an underestimate of the errors, or the presence of unknown systematic errors. It is unlikely that this is due to intrinsic stellar noise as the value of near unity for the reduced $\chi^{2}$ of the HARPS-N RVs implies that the actual errors are consistent with the estimated ones.

Regardless of whether the additional jitter results from systematic errors or stellar intrinsic variations there is no reason to expect that this jitter value should be the same on each night. Rather than applying a single overall jitter correction, we applied a nightly jitter correction term determined in a bootstrap fashion. First, an orbital solution was made using the combined data and nominal errors. The rms scatter of the residuals on a given night was then compared to the mean error. If the actual rms was less than or equal to the mean error, no jitter was added. Otherwise, a jitter correction was added in quadrature with the estimated error in order to make the rms scatter about the orbital fit consistent with the jitter-corrected errors for that night (Table 4). Note that the mean value is consistent with the value of $2.1 \mathrm{~m} \mathrm{~s}^{-1}$ used by H2013. No jitter correction was applied to the HARPS-N data as the reduced $\chi^{2}$ for that data set was already close to unity.

The fit to the combined RV data resulted in $K=1.63 \pm$ $0.23 \mathrm{~m} \mathrm{~s}^{-1}$ and a corresponding planet mass of $1.59 \pm 0.22 M_{\oplus}$. This is Solution 7 in Table 3. The application of the jitter values to the Keck data produced a $\chi^{2}$ of nearly unity. This $K$-amplitude is consistent with both the H2013 and P2013 values with the errors, but our nominal value is closer to the H2013 value.

The lower panel of Fig. 11 shows the L-S periodogram of the RVs after removing the nightly offsets. The dominant peak occurs exactly at the orbital frequency of $v=2.817 \mathrm{~d}^{-1}$. The signal appears to be significant with power, $z=20$ which implies a small false alarm probability $\left(\mathrm{FAP}<10^{-6}\right)$. However, we caution the reader that the FAP may be artificially low since the data has been filtered. In such cases a formal low FAP may not necessarily indicate a high statistical significance (Hatzes 2013).

The FCO technique works best when there are multiple measurements that sample well the segment of the orbit seen on a given night. The Keck RV data, although having a slightly poorer precision, has the advantage over the HARPS-N data of better sampling with 9 to 16 measurements taken each night. We noticed that the fit to the Keck data alone produced a slightly lower $K$-amplitude of $1.30 \pm 0.35 \mathrm{~m} \mathrm{~s}^{-1}$ (Solution 8 in Table 3). This may be due to the fact that the HARPS-N data had fourteen nights on which only two RV measurements were taken. This reflects the strategy by P2013 of taking many measurements at the extrema of the RV curve where one has more "leverage" in terms of detecting a change in the velocity. However, there are two drawbacks to this strategy. First, the RV measurement error is comparable, if not smaller than the $K$-amplitude, so two 


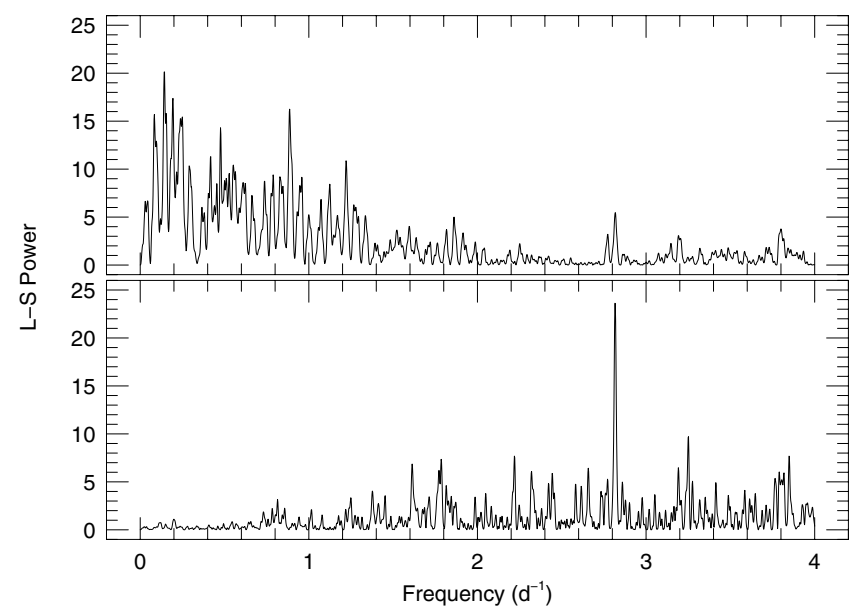

Fig. 11. Top: L-S periodgram of the combined Kepler-78 RVs (HARPS$\mathrm{N}$ plus Keck). An offset has been applied to the HARPS-N data so that both data sets have the same zero-point. Bottom: L-S periodogram of the combined data sets after applying the calculated nightly offsets.

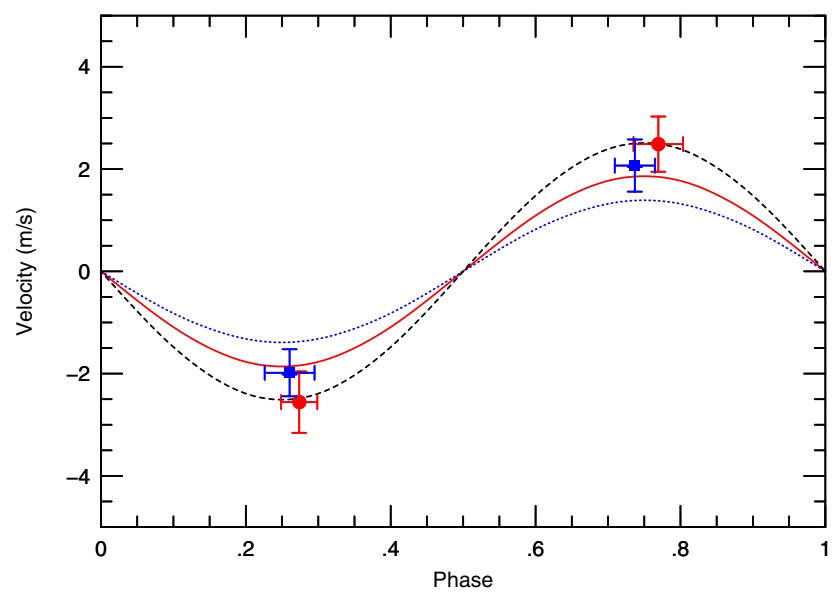

Fig. 12. Phase-binned averages of the orbital variations of Kepler-78b at phases 0.25 and 0.75 , the extrema of the orbital motion. The circles represents values using data from nights were only two RV measurements were made. The squares represent binned averages from nights where typically 6-10 measurements were made. The solid curve represents the orbital solution to the full HARPS-N data set. The dotted line represents the fit using a subset of the HARPS-N measurements where more than two RV measurements on a night were taken. The long-dashed line represents the best fit sine curve with amplitude $K=2.5 \mathrm{~m} \mathrm{~s}^{-1}$ that passes through the RV extrema represented by the circles.

measurements may be insufficient for getting a good RV measurement on a given night. Second, only two measurements in a night provide poor sampling of the shape of the orbital variations. Without the shape information part of the activity related velocity offset may be absorbed into the RV amplitude and could result in a systematicaly higher or lower RV amplitude.

To check this we calculated phase-binned averages around orbital phases 0.25 and 0.75 . We treated the nights with more than two measurements separate to those where only two measurements were made. The binned values from nights with only two measurements show a slightly larger amplitude than that from nights with multiple measurements (Fig. 12). This is also confirmed by calculating an orbit using only the HARPS-N data with more than two measurements per night. The $K$-amplitude is smaller $\left(K=1.39 \pm 0.37 \mathrm{~m} \mathrm{~s}^{-1}\right.$, Solution 9) and is consistent with the amplitude derived using only the Keck data.

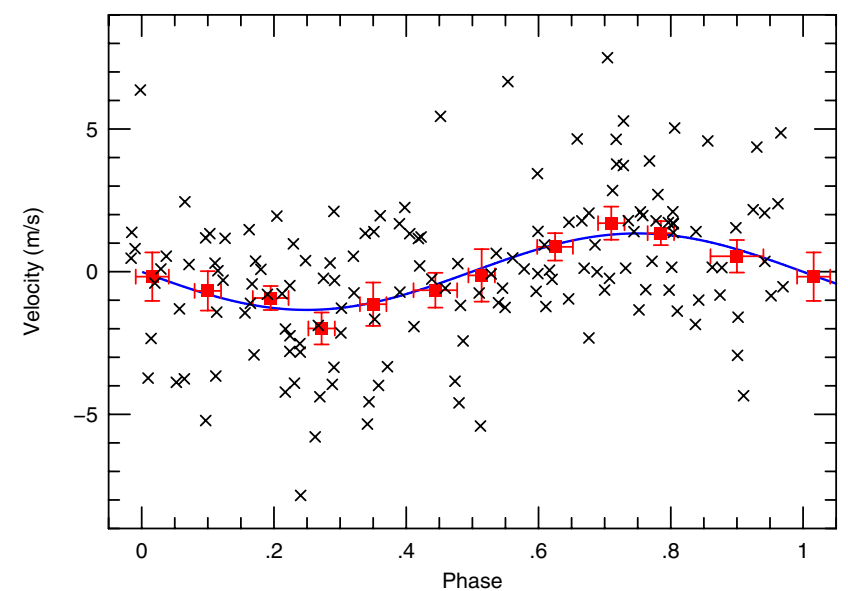

Fig. 13. Phased offset-corrected RV measurements of Kepler-78 RV data for which more than two RV measurements per night were obtained (crosses). The bin-averaged values are shown as squares. The orbital solution is the curve. The "error" on the phase is just the standard deviation of the phases used for the binned value.

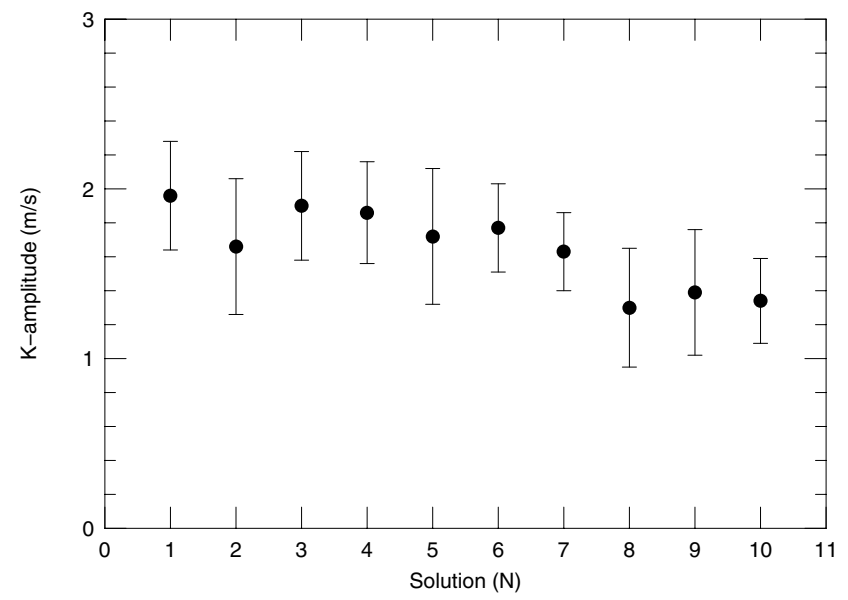

Fig. 14. Various values of the $K$-amplitude using different approaches to removing the activity. The "Solution" number refers are listed in Table 3.

Note that for the HARPS RVs of Kepler-78 the best fitting sine curve that passes through the phase-binned averages at phase 0.25 and 0.75 results in a rather high $K$ amplitude of $2.5 \mathrm{~m} \mathrm{~s}^{-1}$. In this case using only RV data taken at the extrema of the RV curve can result in an estimate of the velocity amplitude that can differ by a factor of two from the value obtained by fitting the full RV curve.

For our "adopted" solution we therefore used only RV data from those nights where more than two RV measurements were taken. This resulted in a $K$-amplitude of $1.34 \pm 0.25 \mathrm{~m} \mathrm{~s}^{-1}$, a corresponding planet mass of $m_{\mathrm{p}}=1.31 \pm 0.24 M_{\oplus}$ (Solution 10), and a density of $\rho=4.5_{-2.0}^{+2.2} \mathrm{~g} \mathrm{~cm}^{3}$ using the planet radius from $\mathrm{P} 2013$. The RV values after subtracting the calculated nightly offsets and phased to the orbital period are shown in Fig. 13. Also shown are phase-binned averages so that one can see better the orbital motion.

All the $K$-amplitude determinations using the various methods including the published values and those from pre-whitening are summarized in Table 3. For all our solutions the period and phase were fixed to the transit values. A graphical version of the table is shown in Fig. 14 where the solution "number" refers to 


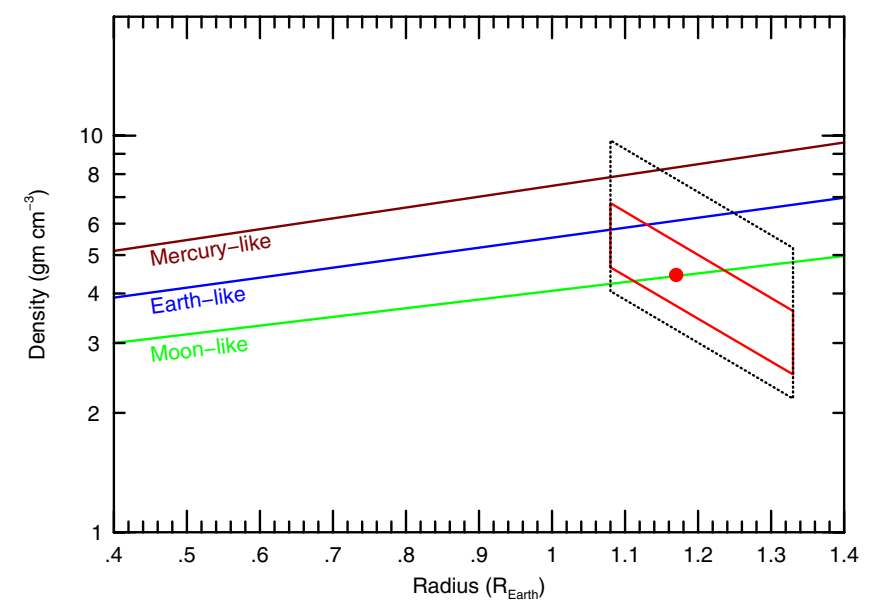

Fig. 15. Density-radius relationships for terrestrial planets having a structure that is Mercury-like (top curve), Earth-like (middle curve) or Moon-like (bottom curve). The large dashed parallelogram marks roughly the range of values from the various mass determinations for Kepler-78b. The point marks are adopted solution and the error (solid parallelogram).

the first entry in the table. We note that all determinations of the $\mathrm{RV}$ amplitude are consistent with each other to within $1 \sigma$.

Although the $K$-amplitude determination is good enough to establish that Kepler-78b is a rocky planet, it is insufficient to establish what type of rocky planet it is. Figure 15 shows the density-radius relationship for terrestrial planets computed by Diana Valencia and shown as Fig. 11 in Hatzes et al. (2010). The dashed parallelogram shows the range of density and radius values for Kepler-78b, including $1 \sigma$ errors, from the various mass determinations. For the planet radius and error we used the value of P2013. The range of possible densities for Kepler-78b encompasses planets that are Mercury-like (i.e. large iron core) to Moon-like (where we define "Moon-like" as having no iron core). Although most density determinations are consistent with an Earth-like structure, the nominal density from our adopted solution implies that Kepler-78b has a structure more like the Moon, i.e. one with a small iron core.

\section{Discussion}

The RV detection of Kepler-78b is robust in spite of the $K$ amplitude being less than the measurement error and at least a factor of five smaller than the amplitude of the activity-related RV jitter. Regardless of the approach one uses to filter out the activity, one arives at the same value for the $K$-amplitude to within $1 \sigma$ (Fig. 14).

In particular, the pre-whitening method seems to provide a good "quick look" method of finding the amplitude of possible signals in RV data. Harmonic filtering where one fits the activity variations with the rotation period and its harmonics is similar to pre-whitening, but with more restrictions on the number and frequency of the components. It is thus not suprising that both give comparable results.

The Fourier amplitude spectrum of the Keck data supports the use of harmonic filtering as the rotational period and its first 3 harmonics are present. This is not the case with the HARPS-N data which shows no clear evidence for either the adopted rotational period or its harmonics. However, the derived RV amplitude seems to be relatively insensitive to the details in how the activity signal is removed.
The RV data for Kepler-78b are so numerous and of such high quality that the planet would have been detected without knowledge of the transit period. In fact, the unfiltered Fourier amplitude spectrum alone indicates the presence of a planet signal with a period of $0.355 \mathrm{~d}$ and a false alarm probability of $\sim 1 \%$. The planet signal planet is significant in the RV data without filtering.

We have shown that the FCO periodogram can be an effective tool for finding unknown short period, low-amplitude planets in RV data that are dominated by activity-related RV variations. In these cases classic periodograms probably would fail. Our simulations show that the FCO periodogram could have discovered a $0.355-\mathrm{d}$ period in the RV data even if the RV amplitude was as low as $K \approx 1 \mathrm{~m} \mathrm{~s}^{-1}$. This corresponds to a planet mass of $1 M_{\oplus}$. This bodes well for finding low mass, ultra-short period planets even in the presence of activity noise.

When extracting the signal of very short period planets in the presence of activity noise we prefer to use the FCO method. It is simple and makes only one reasonable assumption that in the course of the night the RV variations due to planetary orbital motion is much larger than that due to rotational modulation by surface active regions. One needs no knowledge of the rotational period of the planet and we do not care if this changes due to differential rotation. Additionaly, systematic errors on time scales of days or longer are also removed with FCO, as well as the influence of other long period planets that may be in the system (Hatzes et al. 2010). On the other hand, harmonic analysis needs an accurate rotation period, coupled with the assumption that this does not change over the course of the observing season. Furthermore, there is no way to deal with any nightly systematic errors with harmonic analysis or pre-whitening.

The FCO periodogram and offset fitting to the orbit only works, however, if you have 1) a good temporal sampling on a given night; and 2) a planetary orbital period much less than the rotational period of the star which defines the characteristic timescales of activity. Our simulations indicate that if the orbital period of the planet is at least a factor of four shorter than the shortest period associated with activity (the rotation period and its harmonics), the FCO periodogram should be an effective tool for finding unknown short period planets in RV data and may give a better measure of the $K$-amplitude.

When trying to detect RV variations due to low mass planets one may be tempted to just take a few measurements at those times that correspond to the extrema of the RV curve (phases 0.25 and 0.75 for a transiting planet). After all, these should show the maximum RV variation from orbital motion. However, in the presence of activity noise, or when the measurement error dominates the $K$-amplitude of the planet, it may be wiser to simply invest more measurements and sample more of the orbit. After all, there is also information on the $K$-amplitude in the shape of the RV. In the case of Kepler-78b, the orbital solution with all the HARPS-N data yielded a larger $K$-amplitude than the fit to only the Keck data. If one were to only use the RV measurements taken at the expected extrema of the RV curve one would derive an even larger $K$-amplitude $\left(2.5 \mathrm{~m} \mathrm{~s}^{-1}\right)$. A solution using only HARPS-N data where more than two measurements were made per night produced an amplitude consistent with the Keck result. For Kepler-78b this difference is subtle, yet important as it distinguishes whether it has a structure more like the Moon or Mercury.

We know that Kepler-78b is rocky planet, but we do not know what kind of rocky planet it is. The Doppler-determined planet mass depends on the details in how the activity signal is removed as well as having sufficient data. The various 
techniques produced a planet mass (including $1 \sigma$ errors) that ranged from as low as $1.1 M_{\oplus}$ to as high as $2.3 M_{\oplus}$. These yielded bulk densities that were consistent with a Moon-like up to Mercury-like internal structures. The data are insufficient to make the subtle distinction between internal structures, but the precise type of rocky planet may hold the key to understanding how these terrestrial planets form.

It would be possible to distinguish between the types of rocky planet if we had an error of about $5 \%$ on our adopted $K$-amplitude. We estimated the number of measurements that would be required to achieve this accuracy by calculating orbital solutions using a subset of the RV data and sequentially adding more nights into the solution. We estimate that to get an error of $5 \%$ on the $K$-amplitude would require approximately 300 measurements with Keck-like sampling, or about 30 nights of observations. This is a large investment in observing time, but it is important if we are to determine the true structure of Kepler-78b.

Of course, even if we have an excellent measurement of the planet mass with low errors, the density error will be dominated by the errors in the stellar parameters. Fitting the transit light curve only yields the ratio of the panet to stellar radii. Likewise, the velocity $K$-amplitude of the star due to the planet depends on the stellar mass. An accurate planet density requires accurate stellar parameters. This can only be accomplished with an asteroseismic determination of the stellar radius. In this respect the PLATO mission (Rauer et al. 2014) will provide a significant improvement in our understanding of the internal structure of rocky exoplanets. PLATO not only will search for transiting rocky planets around bright stars for which characterization studies are possible, but it will also measure stellar oscillations in order to determine asteroseismic values for the stellar mass and radius.

Acknowledgements. The author would like to thank Malcolm Fridlund for his reading of the manuscript and useful comments.

\section{References}

Hatzes, A. P. 2013a, Astron. Nachr., 334, 616

Hatzes, A. P. 2013b, ApJ, 770, 133

Hatzes, A. P., Dvorak, R., Wuchterl, G., et al. 2010, A\&A, 520, A93

Hatzes, A. P., Fridlund, M., Nachmani, G., et al. 2011, A\&A, 743, A75

Howard, A., Sanchis-Ojeda, R., Marcy, G. W., et al. 2013, Nature, 503, 381 (H2013)

Jefferys, W. H., Fitzpatrick, M. J., \& McArthur, B. E. 1988, Celest. Mech., 41, 39

Kuschnig, R., Weiss, W. W., Gruber, R., Bely, P. Y., \& Jenkner, H. 1997, 328, 544

Lomb, N. R. 1976, Ap\&SS, 39, 447

Pepe, F., Cameron, A. C., Latham, D. W., et al. 2013, Nature, 503, 377 (P2013)

Queloz, D., Bouchy, D., Moutou, C., et al. 2009, A\&A, 506, 303

Rauer, H., Catala, C., Aerts, C., et al. 2014, Exp. Astron., submitted [arXiv: 1310.0696]

Sanchis-Ojeda, R., Rappaport, S., Winn, J. N., et al. 2013, ApJ, 774, 54

Scargle, J. D. 1982, ApJ, 263, 835

Zechmeister, M., \& Kürster, M. 2009, A\&A, 496, 577 